\title{
Internal dynamics of long Josephson junction oscillators
}

Christiansen, Peter Leth; Lomdahl, P. S.; Scott, Alwyn C.; Sørensen, O. H.; Eilbeck, J. C.

\section{Published in:}

Applied Physics Letters

Link to article, DOI:

$10.1063 / 1.92538$

Publication date:

1981

Document Version

Publisher's PDF, also known as Version of record

Link back to DTU Orbit

Citation (APA):

Christiansen, P. L., Lomdahl, P. S., Scott, A. C., Sørensen, O. H., \& Eilbeck, J. C. (1981). Internal dynamics of long Josephson junction oscillators. Applied Physics Letters, 39(1), 108-110. https://doi.org/10.1063/1.92538

\section{General rights}

Copyright and moral rights for the publications made accessible in the public portal are retained by the authors and/or other copyright owners and it is a condition of accessing publications that users recognise and abide by the legal requirements associated with these rights.

- Users may download and print one copy of any publication from the public portal for the purpose of private study or research.

- You may not further distribute the material or use it for any profit-making activity or commercial gain

- You may freely distribute the URL identifying the publication in the public portal 


\section{Internal dynamics of long Josephson junction oscillators}

P. L. Christiansen, P. S. Lomdahl, A. C. Scott, a) and O. H. Soerensen

Laboratory of Applied Mathematical Physics, The Technical University of Denmark, DK-2800 Lyngby,

Denmark.

J. C. Eilbeck

Department of Mathematics, Heriot-Watt University, Edinburgh EH 14 4AS, United Kingdom.

(Received 23 February 1981, accepted for publication 14 March 1981)

Numerical computations on a sine-Gordon model of the Josephson junction fluxon oscillator are compared with experimental measurements. Good agreement is found for the voltage current characteristic, oscillator power output, and range of current bias over which oscillation is observed. Our numerical results imply a "bunched-fluxon" mode of oscillation at larger values of bias current.

PACS numbers: $85.25 .+\mathrm{k}, 74.50 .+\mathrm{r}$

The fluxon oscillator was conceived by Fulton and Dynes ${ }^{1}$ to explain the appearance of zero field steps (ZFS) in the voltage-current $(V-I)$ characteristics of long Josephson junctions, and exploratory measurements of microwave power output have been reported by several authors. ${ }^{2}$ Recent analog ${ }^{3}$ and numerical studies ${ }^{4}$ have suggested that congealed (or "bunched") fluxon states, previously studied in relation to traveling waves, ${ }^{5}$ may play an important role in the dynamics of fluxon oscilllators. This suggestion is supported by very careful measurements made within the past few months ${ }^{6}$ on niobium-lead junctions fabricated in the Department of Physics at the University of Salerno. ${ }^{7}$

In this letter we compare some of these recent measurements with results from a detailed numerical study based upon the (normalized) perturbed sine-Gordon equation ${ }^{8}$

$$
\phi_{x x}-\phi_{t t}-\sin \phi=\alpha \phi_{t}-\beta \phi_{x x t}-\gamma \text {. }
$$

Here $\phi$ is magnetic flux normalized to $\hbar / 2 e, x$ is longitudinal distance normalized to $\lambda_{J}$, and $t$ is time normalized with respect to the Josephson "plasma" frequency $\omega_{0}$. The righthand term in $\alpha$ represents dissipation caused by normal electrons crossing the junction. It is computed as

$$
\alpha=V_{N} /\left(R I_{0}\right),
$$

where $R$ is an effective normal resistance, $I_{0}$ is the maximum zero voltage (Josephson) current, and $V_{N}$ is a normalizing voltage defined by ${ }^{8}$

$$
V=V_{N} \phi_{i} \text {. }
$$

The loss parameter $\beta$ is the reciprocal of the $Q$ of the superconductor surface impedance at $\omega_{0}$ (Ref. 9) and is equal to 0.02 for the niobium-lead junctions considered here. ${ }^{10}$ Finally, $\gamma$ is bias current measured in units of the Josephson current $I_{0}$.

Throughout the computations we have maintained "open-circuit" boundary conditions, i.e., $\phi_{x}(0, t)=\phi_{x}(L, t)=0$, where $L$ is the length of the junction measured in units of $\lambda_{J}$. For the results reported here the initial conditions were two fluxons located at $L / 3$ and $2 L / 3$,

\footnotetext{
"Permanent address: Department of Electrical and Computer Engineering, University of Wisconsin, Madison, Wisconsin 53706, and Center for Nonlinear Studies, Los Alamos National Laboratory, Los Alamos, NM 87545 .
}

thus putting us on the second ZFS. For a fixed number of fluxons the ultimate oscillations were usually independent of the details of the initial conditions, but to obtain fluxon confinement for shorter junctions, it was necessary to launch them with sufficient initial velocity (for example 0.9 on the $L=6$ junction discussed below). We have used an implicit finite difference scheme with a corrector loop for the nonlinear term and averaging of the second space derivative over two time steps. The time and space steps used were 0.05 and 0.1 , respectively. The accuracy of our computations was checked by a systematic halving and doubling of both time and space steps.

Typical numerical results that clearly show the bunched fluxon oscillation mode are presented in Fig. 1." The inset shows $\phi_{t}$ [voltage from Eq. (3)] at one end of the junction for four periods of the oscillation. The numerical integration was carried on until the first three Fourier components of $\phi_{t}(L, t)$ remained constant to within $2 \%$. (For larger values of $\gamma$ this required several hundred thousand time steps.) The zero-order Fourier component (average value) of $\phi_{t}$ corresponds [again through Eq. (3)] to the dc junction voltage. Thus a plot of $\left\langle\phi_{t}\right\rangle V_{N}$ vs $\gamma$, as shown in Fig. 2a,

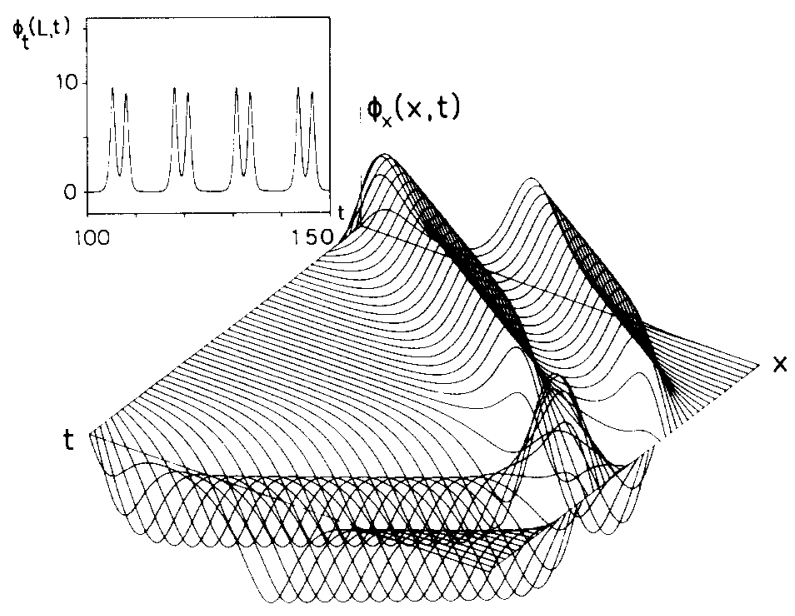

FIG. 1. Bunched-fluxon solution of Eq. (1) with $\alpha=0.05, \beta=0.02$, $\gamma=0.3, L=6$, and two fluxons. Approximately one period of $\phi_{1}(x, t)$ is plotted for $150 \leqslant t \leqslant 160$. The insert shows $\phi,(L, t)$ for $100 \leqslant t \leqslant 150$. 


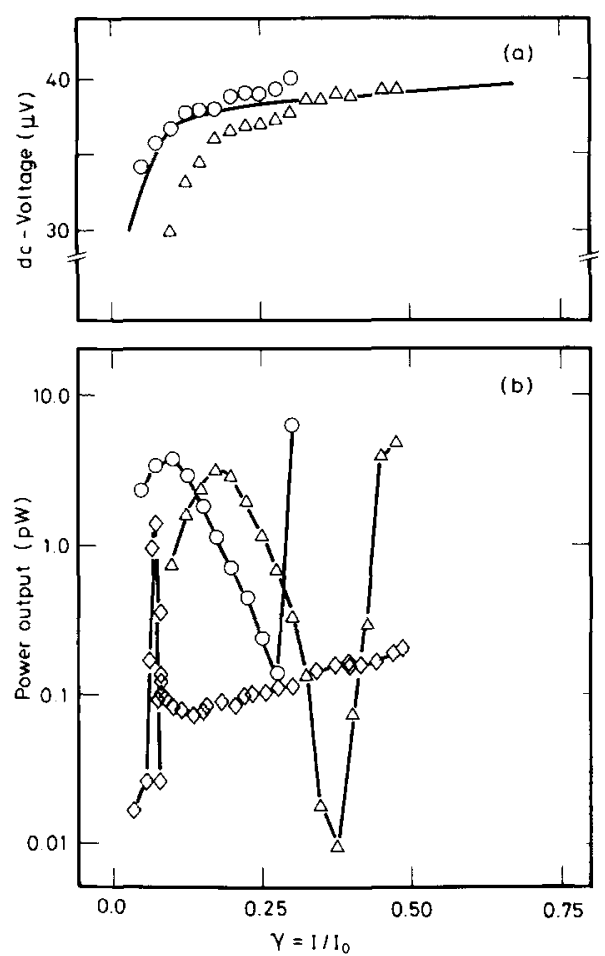

FIG. 2. Comparison of numerical calculations [with $\beta=0.02, L=6$, $\alpha=0.01(0)$, and $\alpha=0.05(\Delta)]$ with measurements on a corresponding niobium-lead Josephson junction oscillator. (a) Average voltage vs current (second zero field step): Measurements (solid line). (b) Power output vs current bias: Measurements (solid-diamond line). Calculated power is into a freespace load of $377 \Omega$.

should correspond to the second ZFS in the $V$ - $I$ characteristic of the junction. Since the normal current across the junction is not proportional to voltage, the effective resistance $[R$ in Eq. (2)] is somewhat difficult to define, but for the particular junction displayed in Fig. 2a, $\alpha$ lies between 0.01 and 0.05 . We see that the lower value of $\alpha$ gives better agreement for lower $\gamma$ and the larger value for larger $\gamma$.

The first harmonic of our numerical computation rises monotonically with $\gamma$. This is not displayed because corresponding measurements are not available. For the second harmonic, however, direct measurements of the output power have been made. ${ }^{2}$ This is plotted in Fig. $2 \mathrm{~b}$ and compared with our numerical calculations. Since the Josephson junction is loosely coupled to the microwave output circuit, ${ }^{12}$ we estimate the output power as (second harmonic component of $\left.\phi_{1}\right)^{2} V_{N}^{2} /$ (twice the free-space impedance). As is noted on Fig. $2 b$, this is the second-harmonic power into $377 \Omega$. There is a fair agreement between calculated and measured levels of output power and the range of $\gamma$ over which two-fluxon oscillation occurs. In addition, both show a pronounced "peak" and "dip" as bias current is changed. From the numerical results it is clear that the dip arises not from a change in mode structure but merely represents the situation in which the fluxon bunches (see inset of Fig. 1) occupy approximately one half-cycle. Thus one should be cautious about interpreting the experimental dip as caused by a mode shift. The numerical results do indicate a change of mode structure from the bunched oscillation shown in Fig. 1 to the symmetric oscillation of Fig. 3, but this change takes place

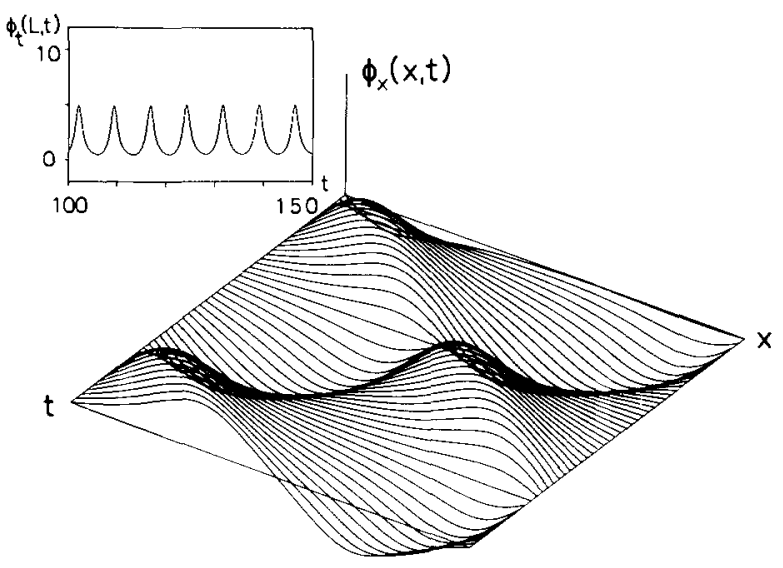

FIG. 3. Symmetric solution of Eq. $(1)$ with $\alpha=0.05, \beta=0.02, \gamma=0.125$, $L=6$, and two fluxons. Approximately one period of $\phi_{r}(x, t)$ is plotted for $150 \leqslant t \leqslant 160$. The inset shows $\phi_{,}(L, t)$ for $100 \leqslant t \leqslant 150$.

gradually as $\gamma$ is reduced and bunched behavior is completely extinguished at the peak rather than the dip. Although the oscillaltion of Fig. 3 is a solution of the perturbed sine-Gordon Eq. (1), it seems most natural to interpret it as a currentdriven cavity mode. Note from the inset of Fig. 3 that the frequency has doubled. Thus it is the first-harmonic power which is plotted on Fig. 2b; this corresponds to the experimental measurements. ${ }^{6.12}$ The differences between calculated and measured locations of the dips and widths of the peaks may be caused by a number of factors including (i) poor representation of the nonlinear conduction current by a linear resistance in Eq. (2); note from Fig. 2(a) that the $V$-I characteristic is not particularly well represented in the low $\gamma$ range; (ii) an underestimate of the effective Josephson current as the maximum zero voltage current. This would lead to an underestimate of the normalized junction length. Since the dip in the second harmonic occurs when the bunch occupies about a half-cycle, longer junctions should show the dip at lower values of bias current.

We are currently exploring the parameter space $(\alpha, \beta, \gamma$, and $L)$ to determine the range of stability for $N$-fluxon oscillations.

It is our pleasure to thank Physics Laboratory I at The Technical University of Denmark and the authors of Ref. 6 for providing the unpublished measurements in Fig. 2 and $\mathbf{R}$. D. Parmentier for many helpful discussions. The support by the Danish Council for Scientific and Technical Research, the Danish Natural Science Research Council, and the Otto M $\phi$ nsteds Fund is acknowledged.

'T. A. Fulton, and R. C. Dynes, Solid State Commun. 12, 57 (1973). ${ }^{2}$ D. N. Langenberg, D. J. Scalapino, and B. N. Taylor, Proc. IEEE 54, 560 (1966). T. A. Fulton, and L. N. Dunkleberger, Rev. Phys. Appl. 9, 299 (1974). T. F. Finnegan, J. Toots, and J. Wilson in Low Temperature Physics, Lett. 14, Vol. 4, edited by M. Krusius and M. Vuorio (North-Holland, Amsterdam, 1975), p. 184.

${ }^{3}$ M. Cirillo, M. Sc. Thesis, University of Salerno, 1979 [in Italian (unpublished)]. M. Cirillo, R. D. Parmentier, B. Savo, Physica $D 1981$ (to be published).

‘'S. N. Erné, R. D. Parmentier, Proceedings of the 1980 Applied Supercon- 
ductor Conference IEEE, Trans, Magn. (to be published). J. Appl. Phys. $51,5025(1980)$

${ }^{5}$ W. J. Johnson, Ph.D. Thesis, University of Wisconsin 1968 (unpublished). See also D. W. McLaughlin and A. C. Scott, Phys. Rev. A 18, 1652 (1978). "B. Dueholm, O. A. Levring, J. Mygind, N. F. Pedersen, O. H. Soerensen, and $M$. Cirillo (unpublished).

'V. Lacquaniti, G. Marullo, and R. Vaglio, IEEE Trans. Magn. MAG-15 $593(1979)$.

${ }^{\times}$A. C. Scott, F. Y. F. Chu, and S. A. Reible, J. Appl. Phys. 47, $3272(1976)$.

"A. C. Scott, Solid-State Electron. 7, 137 (1964). The superconducting films are represented by a distributed circuit model in which each differential section at low frequency is described by a parallel combination of a fre- quency independent resistance $R_{n} d x$ and a frequency-independent inductance $L_{i} d x$. Hence $\beta=\omega_{i} L_{p} / R_{p}=1 / Q$.

"'R. F. Broom and P. Wolf, Phys. Rev. B 16, 3100 (1977). T. Yogi and J. E. Mercereau, Proc. of 1980 Appl. Supercond. Conf. [IEEE Trans. Magn. fto be published)].

"Note that this result is obtained at a junction length $(L=6)$ for which it has previously been suggested (Ref. 4 ) that bunched oscillations do not occur.

${ }^{12}$ B. Dueholm, O. A. Levring, J. Mygind, N. F. Pedersen, O. H. Soerensen, and $\mathrm{M}$. Cirillo (private communication).

\title{
The crystallogaphic orientation of $A 15 \mathrm{~V}_{3} \mathrm{Ga}$ and $\mathrm{Nb}_{3} \mathrm{Al}$ precipitates in $B C C$ matrices
}

\author{
I. -W. Wu, M. Hong, and J. W. Morris, Jr. \\ Materials and Molecular Research Division, Lawrence Berkeley Laboratory, Department of Materials Science \\ and Mineral Engineering, University of California, Berkeley, California 94720
}

(Received 16 February 1981; accepted for publication 6 April 1981)

Convergent-beam transmission electron diffraction was used to determine the crystallographic orientation of submicron-sized $A 15$ structure $\mathrm{V}_{3} \mathrm{Ga}$ and $\mathrm{Nb}_{3} \mathrm{Al}$ precipitates formed by solid-state precipitation from supersaturated $B C C$ solutions. In each case the crystallographic orientation relation was (to within an uncertainty of $\left.\pm 5^{\circ}\right):[001]_{A 15}\left\|[111]_{B C C} ;(100)_{A 15}\right\|(1 \overline{1} 0)_{B C C}$. This lattice correspondence is intuitively plausible, since it ensures that the closest packed planes and directions of the precipitate parallel to those of the matrix.

PACS numbers: 74.70.Lp, 61.50.Jr, 61.14.Fe, 81.10.Jt

Crystalline compounds of the $A 15$ structure are of scientific and engineering interest because of their excellent superconducting properties. The promising $A 15$ compounds are, however, invariably brittle and therefore cannot be directly formed into superconducting wire or tape. $A 15$ superconducting materials are hence usually manufactured in a two-step process, in which wire or tape containing the metallic constituents of the $A 15$ structure is rolled, drawn, or extruded, and the $A 15$ compound is subsequently introduced through an appropriate thermal treatment which causes its precipitation in the solid state. To establish metallurgical control over the processing of $A 15$ superconducting materials it is important to achieve a fundamental understanding of the crystallography, mechanism, and kinetics of the relevant precipitation reactions.

The present investigation was undertaken to determine the common crystallographic relations which govern the orientation of $A 15$ precipitates in $B C C$ media. The two cases specifically studied were $\mathrm{V}_{3} \mathrm{Ga}(A 15)$ in a vanadium-rich V-Ga solid solution $(B C C)$ and $\mathrm{Nb}_{3} \mathrm{Al}(A 15)$ in a nobium-rich $\mathrm{Nb}-\mathrm{Al}$ solid solution $(B C C)$. Samples were made ${ }^{1-3}$ by casting and homogenizing solute-rich metallic solid solutions, deforming these into thin tapes by warm rolling, and aging at intermediate temperature to precipitate the $A 15$ phase. The specific examples illustrated in the accompanying figures are (i) V-18-at. $\% \mathrm{Ga}$, deformed $90 \%$ and aged at $700^{\circ} \mathrm{C}$ for 12 h, and (ii) $\mathrm{Nb}-18$-at. $\% \mathrm{Al}$, deformed $99 \%$ and aged at $750{ }^{\circ} \mathrm{C}$ for $3 \mathrm{~h}$. Thin-foil specimens for transmissions electron microscopic analysis were thinned from these tapes, ${ }^{4}$ and ex- amined through both conventional ${ }^{5}$ and convergent beam ${ }^{6.7}$ electron diffraction analyses to determining the crystallographic relations between the $A 15$ precipitates and the $B C C$ matrix phase.

The analysis of the $\mathrm{V}_{3} \mathrm{Ga}$ precipitate is illustrated in Fig. 1 (a)-(c). The transmission electron micrograph presented in Fig. 1(a) shows the precipitates present in a deformed $\mathrm{V}$-18-at. \% Ga sample after aging at $700^{\circ} \mathrm{C}$ for $12 \mathrm{~h}$. The $\mathrm{V}_{3} \mathrm{Ga}$ precipitates are lenticular in shape and have an average size of $\sim 3000 \AA$ length by $1300 \AA$ width. A typical convergent-beam diffraction pattern from this sample is shown in Fig. 1(b): the disk shape of the diffraction spots is due to the angular convergence of the incident electron beam. ${ }^{6}$ The diffraction pattern is indexed in Fig. 1(c). The zone axes of the $B C C$ matrix and the $A 15$ precipitates are [111] and [001], respectively. The rows of parallel matrix and precipitate spots in the diffraction pattern indicate parallel planes (within $\pm 5^{\circ}$ ), since the corresponding reflecting planes are almost parallel to the electron beam (Bragg angle $\left.<1^{\circ}\right) .^{5}$ It follows that the (100) plane of the $A 15$ precipitate is essentially parallel to the (110) plane of the $B C C$ matrix. The complete orientation relation is hence given by the appealingly simple correspondence:

$$
[001]_{A 15}\left\|[111]_{B C C} ;(100)_{A 15}\right\|(1 \overline{1} 0)_{B C C} .
$$

The analysis of the $\mathrm{Nb}-\mathrm{Al}$ system is illustrated in Fig. 2(a)-2(b). The transmission electron micrograph presented in Fig. 2(a) shows that the $\mathrm{Nb}_{3} \mathrm{Al}$ precipitates formed from $\mathrm{Nb}-18$-at. \% Al on aging at $750^{\circ} \mathrm{C}$ for $3 \mathrm{~h}$ are morphologi- 